\title{
Parametric investigation of InGaAs/InAlAs HEMTs grown by CBE
}

\author{
G.O. Munns, M.E. Sherwin, Y. Kwon, T. Brock, W.L. Chen, D. Pavlidis and G.I. Haddad \\ Center for High Frequency Microelectronics, 1135 EECS Building, The University of Michigan, Ann Arbor, Michigan 48109-2122, USA
}

\begin{abstract}
The InAlAs/InGaAs high electron mobility transistor offers excellent high frequency, low noise operation for amplifiers. While this material system has been grown primarily by conventional MBE, other growth techniques have been examined for improved throughput. The flexibility of chemical beam epitaxy offers semi-infinite sources, good source stability, efficient phosphorus utilization, and extended uptime (more than 560 growth runs over 1.5 years). However, CBE has only recently been shown to produce excellent quality InAlAs suitable for the growth of InAlAs/InGaAs HEMTs [1]. This is the first parametric investigation of the properties of InAlAs/InGaAs HEMTs grown by CBE. A series of lattice matched, pulse doped HEMTs have been grown in which the dopant dose, spacer layer, and channel thickness were systematically varied. Low field $300 \mathrm{~K}$ Hall mobilities as high as $8700 \mathrm{~cm}^{2} / \mathrm{V} \cdot \mathrm{s}$ for a sheet carrier concentration of $3 \times 10^{12} \mathrm{~cm}^{-2}$ have been measured. This mobility is somewhat lower than uniformly doped HEMTs, which have shown mobilities over $10,000 \mathrm{~cm} 2 / \mathrm{V} \cdot \mathrm{s}$ at room temperature. A figure of merit, the low field conductivity, has been correlated among the device structure, gateless saturation currents, and DC and microwave device performance. Its applicability as a rough predictor of device performance will be discussed. For a given spacer thickness, the mobility improves as the pulse dose is decreased up to a mobility somewhat below that for uniformly doped structures. As the dopant to channel thickness is increased, this saturated mobility also increases. Secondary ion mass spectroscopy has shown no increase in carbon or oxygen levels at the dopant pulse. This has led to speculation that interface scattering at the top InAlAs/InGaAs interface may be important; however, initial SIMS results do not conclusively show intermixing of the Group III elements at this interface. It is possible that a reduction in the substrate temperature during growth may improve any interface roughness. Results of this modification in growth conditions shall be reported. Self-aligned $0.15 \mu \mathrm{m}$ HEMTs fabricated from these layers have shown external DC transconductances over $1000 \mathrm{mS} / \mathrm{mm}$, unity current gain cutoff frequencies as high as $190 \mathrm{GHz}$ and unity power gain frequencies above $300 \mathrm{GHz}$. These results and those of more conventional $0.1 \mu \mathrm{m}$ gate length HEMTs demonstrate the potential of InAlAs/InGaAs HEMTs grown by CBE.
\end{abstract}

\section{Introduction}

The InGaAs/InAlAs material system offers several advantages over the GaAs/AlGaAs system for high frequency applications. Higher twodimensional electron gas concentrations, higher peak drift velocities, and higher mobilities in the channel translate into improved microwave and millimeter wave noise and gain performance. Development of pulse doping, undoped caps, superlattice buffers, self-aligned fabrication, and submicron gate lengths have substantially improved device characteristics. This evolution has seen the rise of InAlAs/InGaAs as a technologically important material system.

While MBE has been the primary source of HEMT material, chemical beam epitaxy has recently been shown to be capable of producing excellent quality epi-material [1]. The key to this demonstration has been the development of alternative organometallics to trimethyl aluminum and triethyl aluminum. Such a precursor is trimethyl amine alane, which has less sensitivity to oxygen and has no $\mathrm{Al}-\mathrm{C}$ bonds as do TMAl or TEAl [2]. This source material permits the advantages of CBE to be exploited: the use of semi-infinite sources, good source stability, the lack of oval defects, the flexibility to use phosphorus, and extended vacuum uptime (nearly 600 runs, over $1000 \mu \mathrm{m}$ of material, over $1 \frac{3}{4}$ years).

To show the capability of CBE to grow these structures, a series of pulse doped, lattice matched InGaAs / InAlAs HEMTs with undoped caps has been investigated. This investigation is similar to several studies based on MBE grown material [3]; however, one of the primary goals of this work 
was to establish standard charaterization and correlate it with DC and microwave device performance.

\section{Material growth and device fabrication}

The material for this study was grown in a stock first generation Intevac/Varian CBE system using trimethyl indium, triethyl gallium, trimethyl amine alane, $100 \%$ arsine and phosphine. Conventional solid source silicon was used as the n-type dopant. Optimization of the bulk InAlAs was previously undertaken and has been reported elsewhere [4]. Pulse doped, latticematched InGaAs/InAlAs HEMTs were grown on semi-insulating InP substrate at a temperature of approximately $530^{\circ} \mathrm{C}$. The spacer thickness, dopant dose, and channel thickness were varied to investigate the influence upon Hall characterization, and DC and microwave performance. Two types of HEMTs were subsequently fabricated: (a) standard $0.1 \mu \mathrm{m}$ T-gates with $2 \mu \mathrm{m}$ sourcedrain spacings and (b) $0.15 \mu \mathrm{m}$ self-aligned $\Gamma$ gates with various gate/drain offsets.
The standard T-gate fabrication consists of an optical/e-beam hybrid process: (1) mesa isolation, (2) ohmic definition, (3) e-beam exposure of bilayer resist using side lobes for overhang, (4) wet chemical gate recessing, (5) $\mathrm{Pt} / \mathrm{Ti} / \mathrm{Pt} / \mathrm{Au}$ gate metallization, and (6) interconnect metallization. The $\Gamma$-gate process flow consists of: (1) prealignment marks, gate recess pattern/ deposition and alloying, (2) mesa etching, (3) e-beam definition of $\Gamma$-gate, (4) gate recess etching, (5) $\mathrm{Pt} / \mathrm{Ti} / \mathrm{Pt} / \mathrm{Au}$ gate metallization, (6) ohmic region definition and $\mathrm{Ge} / \mathrm{Au} / \mathrm{Ni} / \mathrm{Ti} / \mathrm{Au}$ deposition and alloying, (7) interconnect metallization. The self-aligned structure was investigated to control the gate to drain distance while maintaining small gate-source spacing. This results in lower output conductance and gate to drain capacitance necessary, both of which are necessary for an increased $f_{\max } / f_{\mathrm{T}}$ [5].

\section{Material characterization}

From initial work performed on both pulse doped and uniformly doped samples and from

Table 1

Correlation table among device structure parameters and DC and microwave characteristics

\begin{tabular}{|c|c|c|c|c|c|c|c|c|c|c|c|c|c|}
\hline $\begin{array}{l}\text { Layer } \\
\text { No. }\end{array}$ & $\begin{array}{l}\text { Channel } \\
\text { thickness } \\
\text { (̊) }\end{array}$ & $\begin{array}{l}\text { Spacer } \\
\text { thickness } \\
(\AA)\end{array}$ & $\begin{array}{l}\mu_{300} \\
\left(\mathrm{~cm}^{2},\right. \\
\mathrm{V} \cdot \mathrm{s})\end{array}$ & $\begin{array}{l}N_{\mathrm{s}} \\
\times 10^{12} \\
\left(\mathrm{~cm}^{-2}\right)\end{array}$ & $\begin{array}{l}\mu_{77} \\
\left(\mathrm{~cm}^{2} /\right. \\
\mathrm{V} \cdot \mathrm{s})\end{array}$ & FOM & $\begin{array}{l}I_{\mathrm{dss}} \\
(\mathrm{mA})\end{array}$ & $\begin{array}{l}G_{\mathrm{m}} \\
\mathrm{SA} \\
(\mathrm{mS} / \\
\mathrm{mm})\end{array}$ & $\begin{array}{l}G_{\mathrm{m}} \\
\mathrm{NSA} \\
(\mathrm{mS} / \\
\mathrm{mm})\end{array}$ & $\begin{array}{l}f_{\mathrm{T}} \\
\mathrm{SA} \\
(\mathrm{GHz})\end{array}$ & $\begin{array}{l}f_{\mathrm{T}} \\
\text { NSA } \\
(\mathrm{GHz})\end{array}$ & $\begin{array}{l}f_{\max } \\
\mathrm{SA} \\
(\mathrm{GHz})\end{array}$ & $\begin{array}{l}f_{\max } \\
\mathrm{NSA} \\
(\mathrm{GHz})\end{array}$ \\
\hline$\overline{502}$ & 400 & 35 & 2077 & 9.00 & 9659 & 2.07 & & & & & & & \\
\hline 510 & 400 & 35 & 8600 & 3.00 & 24000 & 2.58 & & & & & & & \\
\hline 552 & 400 & 35 & 6985 & 3.76 & 16463 & 2.62 & 37 & 827 & & 127 & & 250 & \\
\hline 565 & 200 & 50 & 8751 & 2.60 & 24157 & 2.28 & 22 & 554 & & 100 & & 200 & \\
\hline 593 & 257 & 35 & 6517 & 3.85 & 12439 & 2.51 & & & & & & & \\
\hline 642 & 257 & 35 & 6762 & 3.30 & & 2.23 & & & & & & & \\
\hline 658 & 250 & 35 & 5862 & 4.17 & 11843 & 2.44 & 28 & & & & & & \\
\hline 665 & 350 & 35 & 5342 & 4.50 & 8919 & 2.40 & 38 & & & & & & \\
\hline 666 & 400 & 35 & 6714 & 3.80 & 12046 & 2.55 & & & & & & & \\
\hline 671 & 400 & 50 & 8586 & 3.44 & 23772 & 2.95 & 36 & & 820 & & 167 & & 250 \\
\hline 675 & 400 & 50 & 8019 & 3.68 & 19769 & 2.95 & 42 & 988 & & 187 & & 310 & \\
\hline 675 & & & & & & & 42 & & 880 & & 167 & & 260 \\
\hline 694 & 400 & 50 & 8209 & 1.25 & 26495 & 1.03 & & & & & & & \\
\hline 695 & 400 & 50 & 8171 & 3.70 & 22213 & 3.03 & 35 & & 628 & & 150 & & 220 \\
\hline 774 & 400 & 50 & 8711 & 3.12 & 27288 & 2.72 & 40 & & & & & & \\
\hline 785 & 400 & 50 & 7259 & 3.69 & 17639 & 2.68 & & & & & & & \\
\hline
\end{tabular}

$I_{\mathrm{dss}}$ is for given $45 \mu \mathrm{m}$ wide recess structure; FOM is the figure of merit defined as the product $\mu N_{\mathrm{s}}$;

$G_{\mathrm{m}}$ is the transconductance; $f_{\mathrm{T}}$ and $f_{\max }$ are the unity gain cutoff frequency and unity power gain frequency;

SA refers to self-aligned devices and NSA refers to non-self-aligned devices. 


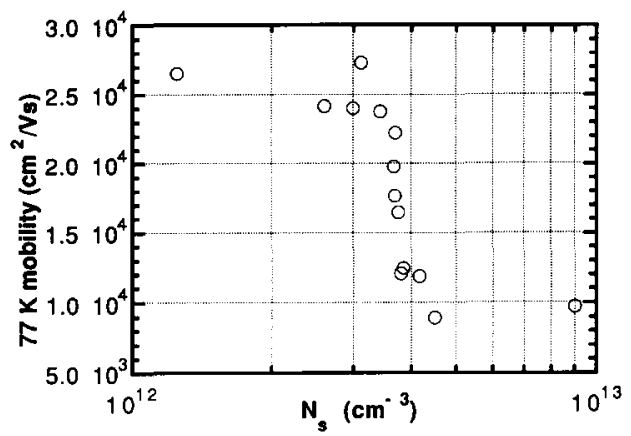

Fig. 1. Mobility at $77 \mathrm{~K}$ versus room-temperature carrier concentration.

first order charge control considerations, it was clear that the sheet carrier concentration should be as high as possible without causing too much scattering, i.e. reduction in Hall mobility at low temperatures. Consequently, Hall measurements were conducted on each sample both at 300 and at $77 \mathrm{~K}$. Table 1 summarizes the structure and device parameter, while fig. 1 shows the $77 \mathrm{~K}$ mobility versus room-temperature carrier concentration. This shows clearly that optimum doping for devices (in this case) lies between 3 and 4 $\times 10^{12} \mathrm{~cm}^{-2}$. While the mobility versus carrier concentration and spacer layer thickness compare favorably to several published reports [6-8], it is also clear that some improvement in these characteristics (structure/growth conditions) might be possible. To this end, the effect of incorporating a superlattice underneath the channel was investigated. Fig. 2 shows SIMS results from this structure. The full width half maximum of the Si

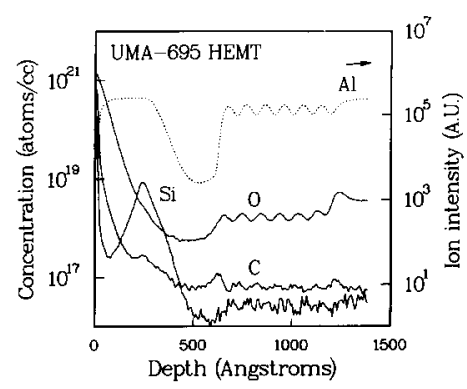

Fig. 2. Secondary ion mass spectra for layer 695 , which incorporated a 5 -period superlattice directly underneath the channel. dopant pulse (100 $\AA$ ) and the asymmetry of the channel region caused concern, despite the indefinite conclusions reached as to the cause of these features. Since it was unclear whether the initial results indicated spreading of the dopant pulse due to temperature or over doping and whether the top InGaAs/InAlAs channel interface was perfectly abrupt, subsequent layers were grown with modified substrate temperature profiles. While the mobility improves in layer \#774, the carrier concentration also decreases. This has been initially attributed to a thicker spacer region due to higher InAlAs growth rate at the lower substrate temperature rather than to smoother interfaces and/or less pulse spreading. Further SIMS studies are underway.

\section{Correlation with device results}

From a simple charge control model, the gateless saturation current should depend only on the sheet carrier concentration

$I_{\mathrm{dss}}=q v_{\mathrm{sat}} N_{\mathrm{s}}$

and the unity current gain cutoff frequency is

$f_{\mathrm{T}}=g_{\mathrm{m}} /\left(2 \pi C_{\mathrm{gs}}\right)=v_{\mathrm{sat}} /(2 \pi L)$,

where $g_{\mathrm{m}}$ is the transconductance, $C_{\mathrm{gs}}$ is the gate/source capacitance, $L$ is the gate length, and $v_{\text {sat }}$ is the saturated velocity. However, the situation for submicron HEMT structures is more complicated and the model used must take into account such additional effects as velocity overshoot/undershoot which are influenced by the low field mobility. Analysis of the results of this study did not show direct linear dependence of microwave characteristics with either the sheet carrier concentration or the low field mobility, individually; however, the low field conductivity, i.e. $q \mu N_{\mathrm{s}}$, did show good agreement with not only microwave performance but also DC characteristics as did the gateless saturation current. Fig. 3 shows the microwave characteristics as a function of the figure of merit ( $\mu N_{\mathrm{s}}$ product). The foundation for this direct proportionality may be found in not only the dependence of the saturation 


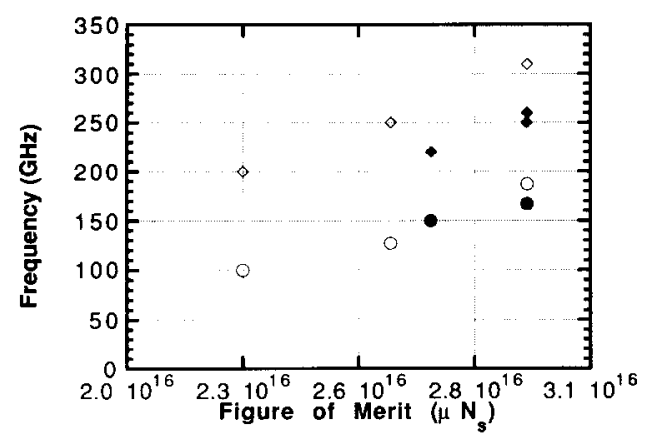

Fig. 3. Unity current gain cutoff frequency $\left(f_{\mathrm{T}}\right)$ and unity power gain cutoff frequency $\left(f_{\max }\right)$ as a function of $\mu^{*} N_{\mathrm{s}}$. Generally accepted $-6 \mathrm{~dB} /$ octave roll-off extrapolation has been used from 1-26 GHz measurements. (O) $f_{\mathrm{T}}$ self-aligned; $(\bullet) f_{\mathrm{T}}$ not self-aligned; $(\diamond) F_{\max }$ self-aligned; $(\bullet) f_{\max }$ not self-aligned.

current on the sheet carrier concentration and the dependence of velocity overshoot on the low field mobility, but also on the access resistance dependence on the marerial conductivity:

$f_{\mathrm{T}}=g_{\mathrm{m}} /\left[2 \pi\left(C_{\mathrm{gs}}+C_{\mathrm{gd}}\right)\left(1+\left(R_{\mathrm{s}}+R_{\mathrm{d}}\right) g_{\mathrm{o}}\right]\right.$,

$f_{\max } \propto f_{\mathrm{T}}$,

where $C_{\mathrm{gd}}$ is the gate feedback capacitance, $R_{\mathrm{s}}$ and $R_{\mathrm{d}}$ are the source and drain resistances, respectively, $g_{\mathrm{m}}$ is the transconductance and $g_{\mathrm{o}}$ is the output conductance. A detailed small signal equivalent circuit model is currently being constructed to determine the limiting parasitics. Fig. 3 also shows the marked improvement in $f_{\max }$ with the self-aligned process in spite of the $50 \%$ longer gate in the self-aligned case.

\section{Conclusions}

Correlating Hall data with DC and microwave performance has shown that the low field con- ductivity $\left(q \mu N_{\mathrm{s}}\right)$ serves as a good relative predictor of submicron device performance. HEMT material grown by $\mathrm{CBE}$ has proven to be comparable to $\mathrm{MBE}$ grown material. Unity power gain cutoff frequencies of self-aligned submicron HEMTs of more than $300 \mathrm{GHz}$ have been realized. The improvement of $f_{\max }$ with self-aligned fabrication has also been demonstrated.

\section{Acknowledgement}

This work was supported by the US Army Research Office, URI Program, Contract DAAL03-86-K-0007.

\section{References}

[1] G.O. Munns, M.E. Sherwin, T. Brock, G.I. Haddad, Y. Kwon, G.I. Ng and D. Pavlidis, J. Crystal Growth 120 (1992) 184.

[2] C.R. Abernathy, A.S. Jordan, S.J. Pearton, W.S. Hobson, D.A. Bohling and G.T. Muhr, Appl. Phys. Letters 56 (1990) 2654.

[3] A.S. Brown, J.K. Mishra, J.A. Henige and M.J. Delaney, J. Vacuum Sci. Technol. B 6 (1988) 678

[4] M.E. Sherwin and G.O. Munns, J. Vacuum Sci. Technol. B 10 (1992) 943.

[5] Y. Kwon, T. Brock, G.I. Ng, D. Pavlidis, G.O. Munns, M.E. Sherwin and G.I. Haddad, presented at 4th Intern. Conf. on InP and Related Materials, Newport, RI, 1991.

[6] F. Gueissaz, R. Houdré and M. Ilegems, J. Crystal Growth 111 (1991) 470.

[7] Y.C. Pao and J.S. Harris, Jr., J. Crystal Growth 111 (1991) 489.

[8] P.C. Chao, A.J. Tessmer, K.G. Duh, P. Ho, M.Y. Kao, P.M. Smith, J.M. Ballingall, S.M.J. Liu and A.A. Jabra, IEEE Electron Device Letters EDL-11 (1990) 59. 